\title{
FROBENIUS COIN-EXCHANGE GENERATING FUNCTIONS
}

\author{
LEONARDO BARDOMERO AND MATTHIAS BECK
}

\begin{abstract}
We study variants of the Frobenius coin-exchange problem: Given $n$ positive relatively prime parameters, what is the largest integer that cannot be represented as a nonnegative integral linear combination of the given integers? This problem and its siblings can be understood through generating functions with 0/1 coefficients according to whether or not an integer is representable. In the 2-parameter case, this generating function has an elegant closed form, from which many corollaries follow, including a formula for the Frobenius problem. We establish a similar closed form for the generating function indicating all integers with exactly $k$ representations, with similar wide-ranging corollaries.
\end{abstract}

\section{THE STORY}

Imagine we replace the penny in the US currency coins by a 7-cent coin. One might argue that the resulting new coin system is a bit less practical than the old, but it is also more (mathematically) interesting: now there are some cent amounts (such as 3 and 8 cents) that cannot be made up using our coins. On the other hand, it is a charming exercise-because 5 and 7 happen to be relatively prime - that every sufficiently large amount of money can be changed; in fact, there are twelve cent amounts that cannot be made up with 5- and 7-cent coins, the largest being 23 cents. (The simple fact that 5 and 7 are relatively prime is crucial—if the greatest common divisor of our coin denominations were $d$, we could not change any amount that is not a multiple of $d$.)

Naturally, nothing stops us (mathematicians) from generalizing this setting, and so for fixed positive relatively prime integers $a_{1}, a_{2}, \ldots, a_{n}$, (that is, $\operatorname{gcd}\left(a_{1}, a_{2}, \ldots, a_{n}\right)=1$ ), we say that a nonnegative integer $x$ is $\left(a_{1}, a_{2}, \ldots, a_{n}\right)$-representable if

$$
x=m_{1} a_{1}+m_{2} a_{2}+\cdots+m_{n} a_{n}
$$

for some $m_{1}, m_{2}, \ldots, m_{n} \in \mathbb{Z}_{\geq 0}$. Let $R_{0}\left(a_{1}, a_{2}, \ldots, a_{n}\right)$ be the set of all positive integers that are not $\left(a_{1}, a_{2}, \ldots, a_{n}\right)$-representable. Because $a_{1}, a_{2}, \ldots, a_{n}$ are relatively prime, $R_{0}\left(a_{1}, a_{2}, \ldots, a_{n}\right)$ is finite, and so three natural questions about this set are:

- What is the largest number $g_{0}\left(a_{1}, a_{2}, \ldots, a_{n}\right)$ in $R_{0}\left(a_{1}, a_{2}, \ldots, a_{n}\right)$ ?

- What is the cardinality $c_{0}\left(a_{1}, a_{2}, \ldots, a_{n}\right)$ of $R_{0}\left(a_{1}, a_{2}, \ldots, a_{n}\right)$ ?

- What is the sum $s_{0}\left(a_{1}, a_{2}, \ldots, a_{n}\right)$ of all elements in $R_{0}\left(a_{1}, a_{2}, \ldots, a_{n}\right)$ ?

The first question is the linear Diophantine problem of Frobenius (it has many alternative names, such as the coin-exchange problem and the chicken nuggets problem), and its solution $g_{0}\left(a_{1}, a_{2}, \ldots, a_{n}\right)$ is called the Frobenius number of the parameter set $\left\{a_{1}, a_{2}, \ldots, a_{n}\right\}$. One of the appealing aspects of the Frobenius problem and its variants is that they can be easily explained. There are many reasons to be interested in the set $R_{0}\left(a_{1}, a_{2}, \ldots, a_{n}\right)$ for fixed $a_{1}, a_{2}, \ldots, a_{n}$; the mathematical basis is the semigroup $S_{0}\left(a_{1}, a_{2}, \ldots, a_{n}\right)$

Date: 13 May 2019, to appear in the American Mathematical Monthly.

2010 Mathematics Subject Classification. Primary 11D07; Secondary 05A15, 05A17.

Key words and phrases. Linear Diophantine problem of Frobenius, coin-exchange problem, Frobenius number, generating function.

We thank Federico Ardila, Yitwah Cheung, two anonymous referees, and the MonTHLY editors for insightful comments about this work. 
generated by $a_{1}, a_{2}, \ldots, a_{n}$, and then $R_{0}\left(a_{1}, a_{2}, \ldots, a_{n}\right)=\mathbb{Z}_{\geq 0} \backslash S_{0}\left(a_{1}, a_{2}, \ldots, a_{n}\right)$. For details about the Frobenius problem, including numerous applications, we recommend two classic MONTHLY articles [15, 23] and the monograph [18].

Our three questions about $R_{0}\left(a_{1}, a_{2}, \ldots, a_{n}\right)$ are, in general, wide open, but they have strikingly simple answers for $n=2$ :

- $g_{0}(a, b)=(a-1)(b-1)-1$;

- $c_{0}(a, b)=\frac{1}{2}(a-1)(b-1)$;

- $s_{0}(a, b)=\frac{1}{12}(a-1)(b-1)(2 a b-a-b-1)$.

The first two formulas go back to at least Sylvester; his paper [21] gives both $c_{0}(a, b)$ and a clear indication that he knew $g_{0}(a, b)$. The third formula is much younger and seems to have first been proved by Brown-Shiue [7]. One can derive all three formulas at once from the following generating function identity.

Theorem 1. Given relatively prime positive integers $a$ and $b$, let $S_{0}(a, b)=\left\{m a+n b: m, n \in \mathbb{Z}_{\geq 0}\right\}$. Then

$$
\sum_{j \in S_{0}(a, b)} z^{j}=\frac{1-z^{a b}}{\left(1-z^{a}\right)\left(1-z^{b}\right)} .
$$

Theorem [1 seems to have first been proved by Székely-Wormald [22] and independently by SertözÖzlük [19]; its usefulness to our three original questions were noticed already in the aforementioned [7]: namely, we observe that

$$
p_{0}(a, b ; z):=\sum_{j \in R_{0}(a, b)} z^{j}=\frac{1}{1-z}-\frac{1-z^{a b}}{\left(1-z^{a}\right)\left(1-z^{b}\right)}
$$

is a polynomial disguised as a rational function, and since

- $g_{0}(a, b)$ equals the degree of $p_{0}(a, b ; z)$,

- $c_{0}(a, b)=\lim _{z \rightarrow 1} p_{0}(a, b ; z)$, and

- $s_{0}(a, b)=\lim _{z \rightarrow 1} p_{0}^{\prime}(a, b ; z)$,

the formulas stated above can be computed by a (patient) calculus student. Theorem 1 is at the heart of this article, and in the interest of self-containment, we will give a proof below. It is a curious fact-and one that is the subject of the MONTHLY papers [8,14] - that we have the alternative form

$$
\sum_{j \in S_{0}(a, b)} z^{j}=\frac{\Phi_{a b}(z)}{1-z},
$$

where $\Phi_{n}(z)$ denotes the $n$th cyclotomic polynomial.

Our goal is to extend the machinery provided by Theorem 1 and its consequences to a recent variant of the Frobenius problem that has attracted some attention in the research community. Namely, we consider the set $R_{k}\left(a_{1}, a_{2}, \ldots, a_{n}\right)$ consisting of all integers with exactly $k$ representations in the form (1), and ask for

- the largest number $g_{k}\left(a_{1}, a_{2}, \ldots, a_{n}\right)$ in $R_{k}\left(a_{1}, a_{2}, \ldots, a_{n}\right)$,

- the cardinality $c_{k}\left(a_{1}, a_{2}, \ldots, a_{n}\right)$ of $R_{k}\left(a_{1}, a_{2}, \ldots, a_{n}\right)$, and

- the sum $s_{k}\left(a_{1}, a_{2}, \ldots, a_{n}\right)$ of all elements in $R_{k}\left(a_{1}, a_{2}, \ldots, a_{n}\right)$.

These are, naturally, hard questions, but there are again answers for $n=2$, both proved in [4]:1]

- $g_{k}(a, b)=(k+1) a b-a-b$

- $c_{k}(a, b)=a b$ for $k \geq 1$.

\footnotetext{
${ }^{1}$ The formula for $c_{k}(a, b)$ appears differently in $[4]$; the difference stems from considering positive vs. nonnegative integers.
} 
Our main contribution is the following generalization of Theorem 1, which will, among other things, allow us to add the missing third bulleted item to the above list.

Theorem 2. Given relatively prime positive integers $a$ and $b$, let $S_{k}(a, b)$ consist of all integers with more than $k$ representations in the form $m a+n b$ with $m, n \in \mathbb{Z}_{\geq 0}$. Then

$$
\sum_{j \in S_{k}(a, b)} z^{j}=\frac{z^{a b k}\left(1-z^{a b}\right)}{\left(1-z^{a}\right)\left(1-z^{b}\right)} .
$$

Consequently, for $k \geq 1$, the polynomial indicating all integers with exactly $k$ representations is

$$
p_{k}(a, b ; z):=\sum_{j \in R_{k}(a, b)} z^{j}=\frac{z^{a b(k-1)}\left(1-z^{a b}\right)^{2}}{\left(1-z^{a}\right)\left(1-z^{b}\right)} .
$$

Naturally, this theorem gives an alternative proof for the above formulas for $g_{k}(a, b)$ (by computing the degree of $p_{k}(a, b ; z)$ ) and $c_{k}(a, b)$ (by computing $\lim _{z \rightarrow 1} p_{k}(a, b ; z)$ ), and because $s_{k}(a, b)=\lim _{z \rightarrow 1} p_{k}^{\prime}(a, b ; z)$, Theorem 2 yields:

Corollary 3. Let $a$ and $b$ be relatively prime positive integers and $k \geq 1$. Then $s_{k}(a, b)=\frac{1}{2} a b(2 a b k-a-b)$.

But Theorem 2 reveals more, namely, that the integers in $R_{k}(a, b)$ (for $k \geq 1$ ) are aligned in a highly structured way, as we may write

$$
p_{k}(a, b ; z)=\sum_{j \in R_{k}(a, b)} z^{j}=z^{a b(k-1)}\left(1+z^{a}+z^{2 a}+\cdots+z^{(b-1) a}\right)\left(1+z^{b}+z^{2 b}+\cdots+z^{(a-1) b}\right) .
$$

Figure 1 illustrates how the sets $R_{k}(a, b)$ are intertwined.

FiguRE 1 . The sets $R_{0}(3,5), R_{1}(3,5), R_{2}(3,5)$, etc.; the shading gets lighter as the index increases.

As an analogue to computing higher moments in statistics, it is natural to ask for higher power sums, or at least their nature. To this extent, we define

$$
s_{k}^{m}\left(a_{1}, a_{2}, \ldots, a_{n}\right):=\sum_{j \in R_{k}\left(a_{1}, a_{2}, \ldots, a_{n}\right)} j^{m}
$$

and offer Theorem 4 below involving the Bernoulli polynomials $B_{n}(x)$, defined as usual through

$$
\frac{z e^{x z}}{e^{z}-1}=\sum_{n \geq 0} \frac{B_{n}(x)}{n !} z^{n}
$$

(see, e.g., [5, Section 2.4]). The first few Bernoulli polynomials are

$$
\begin{aligned}
& B_{0}(x)=1, \\
& B_{1}(x)=x-\frac{1}{2}, \\
& B_{2}(x)=x^{2}-x+\frac{1}{6}, \\
& B_{3}(x)=x^{3}-\frac{3}{2} x^{2}+\frac{1}{2} x, \\
& B_{4}(x)=x^{4}-2 x^{3}+x^{2}-\frac{1}{30}, \\
& B_{5}(x)=x^{5}-\frac{5}{2} x^{4}+\frac{5}{3} x^{3}-\frac{1}{6} x, \\
& B_{6}(x)=x^{6}-3 x^{5}+\frac{5}{2} x^{4}-\frac{1}{2} x^{2}+\frac{1}{42} .
\end{aligned}
$$


The crucial property of Bernoulli polynomials that we will need is (see, e.g., [5, Lemma 2.3])

$$
\beta_{k}(x):=\frac{1}{k}\left(B_{k}(x)-B_{k}(0)\right)=\sum_{j=0}^{x-1} j^{k-1} .
$$

Theorem 4. Let $a$ and $b$ be relatively prime positive integers, $k \geq 1$, and $m \geq 0$. Then

$$
s_{k}^{m}(a, b)=\sum_{\lambda+\mu+v=m}\left(\begin{array}{c}
m \\
\lambda \mu v
\end{array}\right) a^{\lambda+\mu} b^{\lambda+v}(k-1)^{\lambda} \beta_{v+1}(a) \beta_{\mu+1}(b) .
$$

This generalizes the above results for $c_{k}(a, b)$ (which is the case $m=0$ ) and $s_{k}(a, b)$ (the case $m=1$ ), and it gives the asymptotic statement that $s_{k}^{m}(a, b)$ is a polynomial in $k$ of degree $m$ with leading coefficient $(a b)^{m+1}$.

There are other concepts and results hidden in our generating functions. To give a taste, we recall that $S_{k}\left(a_{1}, a_{2}, \ldots, a_{n}\right)$ consists of all integers with more than $k$ representations in the form (1), for general $n$. Thus $\mathbb{Z}_{\geq 0} \backslash S_{k}\left(a_{1}, a_{2}, \ldots, a_{n}\right)$ consists of all nonnegative integers with at most $k$ representations. We define

- $g_{\leq k}\left(a_{1}, a_{2}, \ldots, a_{n}\right)$ as the maximal integer in $\mathbb{Z}_{\geq 0} \backslash S_{k}\left(a_{1}, a_{2}, \ldots, a_{n}\right)$;

- $c_{\leq k}\left(a_{1}, a_{2}, \ldots, a_{n}\right)$ as the cardinality of $\mathbb{Z}_{\geq 0} \backslash S_{k}\left(a_{1}, a_{2}, \ldots, a_{n}\right)$;

- $s_{\leq k}\left(a_{1}, a_{2}, \ldots, a_{n}\right)$ as the sum of all elements in $\mathbb{Z}_{\geq 0} \backslash S_{k}\left(a_{1}, a_{2}, \ldots, a_{n}\right)$.

In words, $g_{\leq k}\left(a_{1}, a_{2}, \ldots, a_{n}\right)$ is the largest integer with at most $k$ representations, $c_{\leq k}\left(a_{1}, a_{2}, \ldots, a_{n}\right)$ is the number of integers with at most $k$ representations, and $s_{\leq k}\left(a_{1}, a_{2}, \ldots, a_{n}\right)$ is the sum of all integers with at most $k$ representations.

The following result can be proved directly from the first part of Theorem 2. (We note that the formulas for $g_{\leq k}(a, b)$ and $c_{\leq k}(a, b)$ are not new.)

Corollary 5. Let $a$ and $b$ be relatively prime positive integers and $k \geq 0$. Then

- $g_{\leq k}(a, b)=(k+1) a b-a-b$;

- $c_{\leq k}(a, b)=\frac{1}{2}(a-1)(b-1)+a b k$;

- $s_{\leq k}(a, b)=\frac{1}{2} a^{2} b^{2} k^{2}+\frac{1}{2}(a b-a-b) a b k+\frac{1}{6} a^{2} b^{2}-\frac{1}{4}(a+b-1) a b+\frac{1}{12}\left(a^{2}+b^{2}-1\right)$.

We remark that $g_{\leq k}\left(a_{1}, a_{2}, \ldots, a_{n}\right)=g_{k}\left(a_{1}, a_{2}, \ldots, a_{n}\right)$ holds only for $n=2$; in fact, for general $n$ these two invariants can differ quite a bit [3,20].

\section{PROOFS}

Proof of Theorem 1 Let

$$
r(a, b ; j):=\left|\left\{(m, n) \in \mathbb{Z}_{\geq 0}^{2}: m a+n b=j\right\}\right|,
$$

the number of representations of $j$ in terms of $a$ and $b$. By a simple geometric series argument,

$$
\sum_{j \geq 0} r(a, b ; j) z^{j}=\frac{1}{\left(1-z^{a}\right)\left(1-z^{b}\right)} .
$$

We claim that

$$
r(a, b ; j) \leq 1 \text { for } j<a b \quad \text { and } \quad r(a, b ; j)=r(a, b ; j-a b)+1 \text { for } j \geq a b,
$$

and so, in particular, any integer $\geq a b$ belongs to $S_{0}(a, b)$. There are several ways to prove (5), for example, by considering the set

$$
M_{j}:=\left\{m \in \mathbb{Z}_{\geq 0}: m a+n b=j \text { for some } n \in \mathbb{Z}_{\geq 0}\right\} .
$$

Then $M_{j} \subset\left[0, \frac{j}{a}\right]$ (because $m, n \in \mathbb{Z}_{\geq 0}$ ), and indeed, if $m \in M_{j}$, then $M_{j}=\left[0, \frac{j}{a}\right] \cap(m+b \mathbb{Z}$ ), which follows from basic number theory (and here the condition $\operatorname{gcd}(a, b)=1$ is vitally important). Thus, for $j<a b$, the set 
$M_{j}$ contains at most one element. For $j \geq a b$, we have the implication $m \in M_{j-a b} \Longrightarrow m \in M_{j}$, by replacing $n$ with $n+a$. Moreover, the set difference $M_{j} \backslash M_{j-a b}=\left(\frac{j}{a}-b, \frac{j}{a}\right] \cap(m+b \mathbb{Z})$ contains precisely one point, and (5) follows.

By (5),

$$
\sum_{j \in S} z^{j}=\sum_{j=0}^{a b-1} r(a, b ; j) z^{j}+\sum_{j \geq a b}(r(a, b ; j)-r(a, b ; j-a b)) z^{j}=\left(1-z^{a b}\right) \sum_{j \geq 0} r(a, b ; j) z^{j} .
$$

Theorem 1 follows now with (4).

Proof of Theorem 2 We proceed by induction on $k$; the base case is Theorem 1 . For the induction step, assume that

$$
\sum_{j \in S_{k-1}(a, b)} z^{j}=\frac{z^{a b(k-1)}\left(1-z^{a b}\right)}{\left(1-z^{a}\right)\left(1-z^{b}\right)} .
$$

Now (5) implies for $j \geq a b$ and $k \geq 1$

$$
j \in S_{k}(a, b) \quad \Longleftrightarrow \quad j-a b \in S_{k-1}(a, b)
$$

(we stress once more that this heavily depends on $a$ and $b$ being relatively prime), and so by induction hypothesis,

$$
\begin{aligned}
\sum_{j \in S_{k}(a, b)} z^{j} & =\sum_{j \in S_{k-1}(a, b)} z^{j+a b} \\
& =z^{a b} \frac{z^{a b(k-1)}\left(1-z^{a b}\right)}{\left(1-z^{a}\right)\left(1-z^{b}\right)} \\
& =\frac{z^{a b k}\left(1-z^{a b}\right)}{\left(1-z^{a}\right)\left(1-z^{b}\right)} .
\end{aligned}
$$

The formula for $p_{k}(a, b ; z)$ now follows from the fact that $R_{k}(a, b)=S_{k-1}(a, b) \backslash S_{k}(a, b)$.

Proof of Theorem 4 We start by noting that the operator $\Delta:=z \frac{d}{d z}$ is very useful in studying our power sums, as

and thus

$$
\Delta z^{j}=z \frac{d}{d z} z^{j}=j z^{j}
$$

$$
s_{k}^{m}(a, b)=\lim _{z \rightarrow 1} \Delta^{m} p_{k}(a, b ; z) .
$$

The operator $\Delta$ satisfies the same product rule as the derivative, and so by [2],

$$
\Delta^{m}\left(p_{k}(a, b ; z)\right)=\sum_{\lambda+\mu+v=m}\left(\begin{array}{c}
m \\
\lambda \mu \nu
\end{array}\right) \Delta^{\lambda}\left(z^{a b(k-1)}\right) \Delta^{\mu}\left(\sum_{j=0}^{b-1} z^{j a}\right) \Delta^{v}\left(\sum_{j=0}^{a-1} z^{j b}\right)
$$

and thus

$$
\begin{aligned}
s_{k}^{m}(a, b) & =\sum_{\lambda+\mu+v=m}\left(\begin{array}{c}
m \\
\lambda \mu
\end{array}\right)(a b(k-1))^{\lambda}\left(\sum_{j=0}^{b-1}(j a)^{\mu}\right)\left(\sum_{j=0}^{a-1}(j b)^{v}\right) \\
& =\sum_{\lambda+\mu+v=m}\left(\begin{array}{c}
m \\
\lambda \mu
\end{array}\right) a^{\lambda+\mu} b^{\lambda+v}(k-1)^{\lambda}\left(\sum_{j=0}^{b-1} j^{\mu}\right)\left(\sum_{j=0}^{a-1} j^{v}\right) .
\end{aligned}
$$

We finish by substituting for the expressions in the last two parentheses using (3). 


\section{Musings ABOUT $n \geq 3$}

The reader might have noticed the striking similarities between the rational generating function in Theorem 1 and that in (4); however, this is an artifact of the case $n=2$. While it is true that the general counting function

$$
r\left(a_{1}, a_{2}, \ldots, a_{n} ; j\right):=\left|\left\{\mathbf{m} \in \mathbb{Z}_{\geq 0}^{n}: m_{1} a_{1}+m_{2} a_{2}+\cdots+m_{n} a_{n}=j\right\}\right|
$$

comes with the generating function

$$
\sum_{j \geq 0} r\left(a_{1}, a_{2}, \ldots, a_{n} ; j\right) z^{j}=\frac{1}{\left(1-z^{a_{1}}\right)\left(1-z^{a_{2}}\right) \cdots\left(1-z^{a_{n}}\right)}
$$

and also that

$$
\sum_{j \in S_{0}\left(a_{1}, a_{2}, \ldots, a_{n}\right)} z^{j}=\frac{h(z)}{\left(1-z^{a_{1}}\right)\left(1-z^{a_{2}}\right) \cdots\left(1-z^{a_{n}}\right)}
$$

for some polynomial $h(z)$, the form of $h(z)$ is simple only for $n \leq 2$. At any rate, Denham [10] discovered the remarkable fact that for $n=3$, the polynomial $h(z)$ has either 4 or 6 terms. He gave semi-explicit formulas for $h(z)$, from which one can deduce a semi-explicit formula for the Frobenius number $g_{0}\left(a_{1}, a_{2}, a_{3}\right)$. This formula was independently found by Ramírez-Alfonsín [17]. Denham's theorem implies that the Frobenius number in the case $n=3$ is quickly computable, which was previously known [9,11,12]. Bresinsky [6] proved that for $d \geq 4$, there is no absolute bound for the number of terms in $h(z)$, in sharp contrast to Denham's theorem.

On the computational side, Barvinok-Woods [2] proved that for fixed $n$, the rational generating function (6) can be written as a short sum of rational functions; in particular, (6) can be efficiently computed when $n$ is fixed. A corollary of this fact is that the Frobenius number can be efficiently computed when $n$ is fixed, a theorem originally due to Kannan [13]. The analogous result for the generalized Frobenius numbers $g_{k}\left(a_{1}, a_{2}, \ldots, a_{n}\right)$ is due to Aliev-De Loera-Louveaux [1]. On the other hand, Ramírez-Alfonsín [16] proved that trying to efficiently compute the Frobenius number is hopeless if $n$ is left as a variable.

As a final note, while our results give a clear picture what kind of functions to expect for $n=2$-e.g., $s_{k}(a, b)$ is linear in $k$ and $s_{\leq k}(a, b)$ is quadratic in $k$-it is unclear to us how this generalizes to $n \geq 3$. Some basic structural results would undoubtedly shed new light on generalized Frobenius numbers and their relatives.

\section{REFERENCES}

1. Aliev, I., De Loera, J. A., Louveaux, Q. (2016). Parametric polyhedra with at least $k$ lattice points: their semigroup structure and the $k$-Frobenius problem. Beveridge, A., Griggs, J. R., Hogben, L., Musiker, G., Tetal, P., eds. Recent Trends in Combinatorics. IMA Volumes in Mathematics 159. Cham: Springer, pp. 753-778.

2. Barvinok, A., Woods, K. (2003). Short rational generating functions for lattice point problems. J. Amer. Math. Soc. 16(4): 957-979.

3. Beck, M., Kifer, C. (2011). An extreme family of generalized Frobenius numbers. Integers. 11(A24): 6 pp.

4. Beck, M., Robins, S. (2004). A formula related to the Frobenius problem in two dimensions. Chudnovsky, D., Chudnovsky, G., Nathanson, M., eds. Number theory (New York, 2003). New York: Springer, pp. 17-23.

5. Beck, M., Robins, S. (2015). Computing the Continuous Discretely: Integer-point Enumeration in Polyhedra. 2nd ed. New York: Springer.

6. Bresinsky, H. (1975). Symmetric semigroups of integers generated by 4 elements. Manuscripta Math. 17(3): $205-219$.

7. Brown, T. C., Shiue, P. J.-S. (1993). A remark related to the Frobenius problem. Fibonacci Quart. 31(1): 32-36.

8. Carlitz, L. (1966). The number of terms in the cyclotomic polynomial $F_{p q}(x)$. Amer. Math. Monthly. 73: 979-981.

9. Davison, J. L. (1994). On the linear Diophantine problem of Frobenius. J. Number Theory. 48(3): 353-363.

10. Denham, G. (2003). Short generating functions for some semigroup algebras. Electron. J. Combin. 10(36): 7 pp.

11. Greenberg, H. (1980). An algorithm for a linear Diophantine equation and a problem of Frobenius. Numer. Math. 34(4): 349-352.

12. Herzog, J. (1970). Generators and relations of abelian semigroups and semigroup rings. Manuscripta Math. 3: $175-193$. 
13. Kannan, R. (1992). Lattice translates of a polytope and the Frobenius problem. Combinatorica. 12(2): 161-177.

14. Moree, P. (2014). Numerical semigroups, cyclotomic polynomials, and Bernoulli numbers. Amer. Math. Monthly. 121(10): 890-902.

15. Nijenhuis, A. (1979). A minimal-path algorithm for the "money changing problem." Amer. Math. Monthly. 86(10): 832-835.

16. Ramírez-Alfonsín, J. L. (1996). Complexity of the Frobenius problem. Combinatorica. 16(1): 143-147.

17. Ramírez-Alfonsín, J. L. (2002). The Frobenius number via Hilbert series. Preprint.

18. Ramírez-Alfonsín, J. L. (2005). The Diophantine Frobenius Problem. Oxford Lecture Series in Mathematics and its Applications, vol. 30. Oxford: Oxford Univ. Press.

19. Sertöz, S., Özlük, A. E. (1991). On the number of representations of an integer by a linear form. Istanbul Üniv. Fen Fak. Mat. Derg. 50: 67-77.

20. Shallit, J., Stankewicz, J. (2011). Unbounded discrepancy in Frobenius numbers. Integers. 11(A2): 8 pp.

21. Sylvester, J. J. (1884). Mathematical questions with their solutions. Educational Times. 41: 171-178.

22. Székely, L. A., Wormald, N. C. (1986). Generating functions for the Frobenius problem with 2 and 3 generators. Math. Chronicle. 15: 49-57.

23. Wilf, H. S. (1978). A circle-of-lights algorithm for the "money-changing problem." Amer. Math. Monthly. 85(7): 562-565.

Department of Mathematics, San Francisco State University, San Francisco, CA 94132, U.S.A.

E-mail address: [josebardomero, becksf su] @gmail.com 